\title{
Tumor associated mast cells: biological roles and therapeutic applications
}

\author{
Shikha Saxena ${ }^{1}$, Anil Singh ${ }^{2}$, Priyanka Singh ${ }^{3}$ \\ ${ }^{1}$ Department of Oral Pathology, RUHS College of Dental Sciences (Government Dental College), Jaipur, Rajasthan, ${ }^{2}$ Department of Oral Pathology, \\ Saraswati Dental College and Hospital, Lucknow, Uttar Pradesh, ${ }^{3}$ Department of Oral Pathology, Faculty of Dental Sciences, King George Medical \\ University, Lucknow, Uttar Pradesh, India
}

\begin{abstract}
Mast cells (MCs) are immune cells of the myeloid lineage and are present in connective tissues throughout the body. The activation and degranulation of MCs significantly modulates many aspects of physiological and pathological conditions in various settings. Recent data have expanded the concept that inflammation is a critical component for tumor progression. Interestingly, three of the most aggressive human cancers, malignant melanoma, breast carcinoma and colorectal adenocarcinoma, are commonly associated with a marked host response comprising of various inflammatory cells, but especially MCs around the tumor periphery. A systematic review of the literature was performed based on the English titles listed in the PubMed, EBSCO, Cochrane, Science Direct, ISI web Science, and SciELO databases using the keywords. Abstracts and full-text articles were assessed. This review summarizes the current understanding of the role of MCs in tumor progression.
\end{abstract}

Key words: Mast cell activation, Development, Proliferation, Migration, Survival

Received August 16, 2019; Revised July 17, 2020; Accepted August 5, 2020

\section{Introduction}

Mast cells (MCs) are ubiquitously distributed, resident connective tissue cells. They are particularly frequent in close proximity to epithelial surfaces in the skin, the respiratory system and the gastrointestinal mucosa where they are strategically located for optimal interaction with the environment and for their putative function in host defence. MCs secrete wide range of proinflammatory, immune-modulatory and mitogenic cytokines [1].

MCs possess many properties that enable them to participate in a diverse range of biological activities. They phagocytoze, process antigens, produce cytokines and release a va-

\section{Corresponding author:}

Shikha Saxena (iD)

Department of Oral Pathology, RUHS College of Dental Sciences (Government Dental College), Jaipur, Rajasthan 302017, India

E-mail: drshikhasaxena29@gmail.com riety of preformed mediators (e.g., histamine, proteoglycans and proteases) and newly formed physiological mediators (e.g., leukotrienes [LTs] and prostaglandins). MCs carry an array of adhesion molecules, immune response receptors and other surface molecules, which permit them to react to multiple specific and nonspecific stimuli. These wide-ranging biological characteristics, their ubiquitous distribution and strategic location near blood vessels, nerves, inflamed tissues and neoplastic foci enable them to play a central role in a multitude of physiologic, immunologic and pathologic processes [2].

Recently, apart from their roles in the maintenance of homeostasis and in inflammation, the association of MCs with various tumours has been described. In several malignancies, mast cell density (MCD) has been found to correlate with increased risk of metastasis and poor prognosis. Currently, the exact functional relevance of MCs surrounding various tumours is being studied [3].

A systematic review of the literature was performed

\section{Copyright $@ 2020$. Anatomy \& Cell Biology}

This is an Open Access article distributed under the terms of the Creative Commons Attribution Non-Commercial License (http://creativecommons.org/licenses/by-nc/4.0/) which permits unrestricted non-commercial use, distribution, and reproduction in any medium, provided the original work is properly cited. 
based on the English titles listed in the PubMed, EBSCO, Cochrane, Science Direct, ISI web Science, and SciELO databases using the keywords. Abstracts and full-text articles were assessed.

\section{Historical Background}

Galli [4] in their review article mentioned that Paul Ehrlich (1877) was the first to describe cells in connective tissue that stained reddish purple (metachromasia) with aniline dyes. He used the term 'mastzellen' to describe these cells, a German term referring to feeding [4].

Galli [5] suggested that MC can also stand for master cell because of their wide anatomical distribution, sensitivity to activation by different stimuli and their ability to release a variety of mediators including cytokines.

\section{Mast Cell Development}

\section{Mast cells arise from multipotent hematopoietic progenitors in the bone marrow}

During the first 100 years after Paul Ehrlich discovered them, MCs were believed to be a component of connective tissue that was derived from undifferentiated mesenchymal cells. However, recent studies have demonstrated that MCs arise from multipotent hematopoietic progenitors in bone marrow [6]. The human MCs originate from CD34+ human progenitor cells and require additional conditions in vitro for the development of MC granule structure [7].

MCs normally do not mature before leaving the bone marrow but circulate through the vascular system as immature progenitors that then complete their development peripherally within connective or mucosal tissues. MC development and growth are crucially regulated by the survival and developmental factor, stem cell factor (SCF). SCF is a pivotal growth factor that promotes the development of human MCs (Table 1) $[8,9]$.

\section{Types of Mast Cells}

\section{Connective tissue mast cells}

Found in skin, peritoneal cavity and muscularis propria of stomach. These cells predominantly contain heparin and large amounts of histamine, can be detected in formalin fixed tissues. Cytoplasm is densely packed with intensely violet, metachromatic granules.

\section{Mucosal mast cells}

Seen in respiratory tract and lamina propria of gastrointestinal mucosa. They predominantly contain chondroitin sulfate, lack heparin and have small amounts of histamine. The cell is smaller, more sparsely granulated and stains reddish with basic dyes [10].

Descriptive classification based on contents of cytoplasmic granules and anatomic localization of cells [11]:

- $\mathrm{MC}_{\mathrm{T}}$ : MCs contain only tryptase, demonstrate scrolllike granules electron microscopically and predominate in intestinal mucosa and lung parenchyma [1].

- $\mathrm{MC}_{\mathrm{TC}}$ : $\mathrm{MCs}$ express both tryptase and chymase along with carboxypeptidase and cathepsin G. It predominates in the gastrointestinal tract as well as in skin, synovium and subcutaneous tissues [1].

$-\mathrm{MC}_{\mathrm{C}}$ : MCs with granules containing chymase without tryptase and predominate in skin, lymph nodes and intestinal submucosa [11].

\section{Structural Aspects of the Human Mast Cell}

On light microscopy, using special stains like toluidine blue and Giemsa, MCs can be appreciated as large cells with a diameter varying from $5-15 \mu \mathrm{m}$ exhibiting heterogeneity

Table 1. The table below lists the growth factors and cytokines that are involved in MC growth and differentiation in human systems [9]

\begin{tabular}{|c|c|c|}
\hline Cytokine & Receptor & Effect on human MCs \\
\hline SCF & Kit & $\begin{array}{l}\text { Directly stimulates proliferation of committed } \\
\text { progenitors } \\
\text { Induces granulation and connective tissue pro- } \\
\text { teases }\end{array}$ \\
\hline IL-3 & IL-3R & $\begin{array}{l}\text { Directly stimulates proliferation of uncommitted } \\
\text { progenitors } \\
\text { No promotion of granule assembly }\end{array}$ \\
\hline IL-4 & IL-4R & Depends on the MC subtype or cytokine milieu \\
\hline IL-5 & IL-5R & Co-factor for proliferation \\
\hline IL-6 & IL-6R & Co-factor for proliferation or inhibition \\
\hline IL-9 & IL-9R & Co-factor for proliferation \\
\hline IL-10 & IL-10R & Not clearly characterized \\
\hline IFN- $\gamma$ & INF- $\gamma \mathrm{R}$ & Inhibits proliferation \\
\hline NGF & NGFR & Inhibits apoptosis in the presence of SCF \\
\hline TGF- $\beta$ & TGF $\beta$ R & Inhibits proliferation \\
\hline GM-CSF & GM-CSFR & Inhibits proliferation \\
\hline TPO & TPOR & Induces MC development \\
\hline
\end{tabular}

MC, mast cell; SCF, stem cell factor; IL, interleukin; IFN, interferon; NGF, nerve growth factor; TGF, transforming growth factor; GM-CSF, granulocytemacrophage colony stimulating factor; TPO, thrombopoietin. 


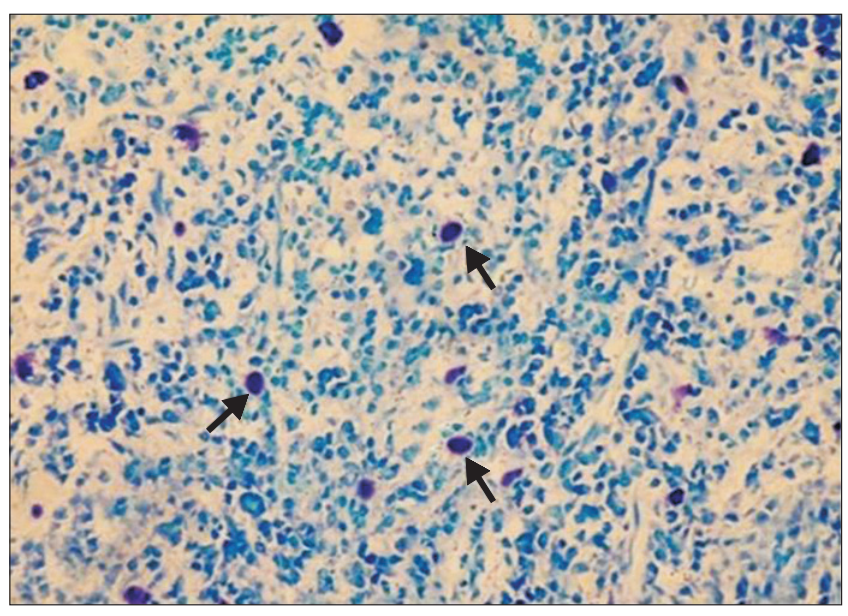

Fig. 1. Photomicrograph of toluidine blue stained section showing mast cells in the human oral mucosal connective tissue $(40 \times)$.

in shape from round or oval to spindle shaped. The nucleus is small, inconspicuous and ovoid in shape and is often masked by cytoplasmic granules. The cytoplasm contains numerous coarse and dense granules, which are refractile, water soluble and exhibit metachromasia, varying in number from 50-200 (0.1-0.4 $\mu \mathrm{m}$ in diameter) (Fig. 1) [12].

\section{Mast Cell Distribution}

MCs are normally distributed throughout the connective tissue, where they may be especially numerous beneath the epithelial surfaces of the skin, in the respiratory system, in the gastrointestinal and genitourinary tracts, adjacent to blood or lymphatic vessels and near or within the peripheral nerves [4].

\section{Activation of Mast Cells and Degranulation}

The MC is a specialized secretory cell exhibiting surface expression of high affinity IgE receptor $\left(\mathrm{F}_{\mathrm{C}} \varepsilon \mathrm{RI}\right)$. When MCs are exposed to specific multivalent antigen or immune complexes, crosslinking of $\mathrm{F}_{\mathrm{C}} \varepsilon \mathrm{RI}$ results in an integrated non cytolytic series of events termed 'activation' [13].

\section{Mast Cell Mediators}

MCs can elaborate and secrete several biologically active compounds. Mediator release can be induced by a variety of stimuli including specific antigens, complement products, neuropeptides and many physical agents [14].
MCs both release and generate a heterogeneous group of mediators that differ in their potency and biological activities. These mediators are both pleiotropic and redundant; that is, each mediator has more than one function and mediators may overlap in their biological effects. MC-dependent mediators may be categorized into three groups: preformed secretory granule-associated mediators, lipid-derived mediators and cytokines [15].

\section{Granule-associated mediators}

\section{Histamine}

Histamine is the single amine known to be stored in human MCs. Storage of histamine in secretory granules is associated by ionic linkage with the carboxyl groups of proteins and proteoglycans of the secretory granules at acidic $\mathrm{pH}$ [15].

\section{Proteoglycans}

The proteoglycans heparin and chondroitin sulfate $\mathrm{E}$ have been associated with human MCs. Both heparin and chondroitin sulfate E stabilize MC proteases and alter the biological activity of many enzymes. Heparin is also a potent anticoagulant [15].

Neutral proteases

Human MCs contain three classes of proteases

- Carboxypeptidase A

- Chymase

- Tryptase

Carboxypeptidase A is stored in the MC granule complexed with proteoglycans. Human MC carboxypeptidase is associated with the $\mathrm{MC}_{\mathrm{TC}}$ type of $\mathrm{MC}$ [16].

Human MC chymase is present in $85 \%$ of the MCs of the skin and intestinal submucosa. It converts angiotensin I to II, may degrade the basement membrane at the dermalepidermal junction, stimulates mucus secretion, degrades neuropeptides and converts a precursor of IL- $1 \beta$ to an active form [17].

Tryptase is the predominant enzyme and is associated with all human MCs examined. It cleaves fibrinogen, activates latent collagenase, hydrolyzes some neuropeptides and may cause mucus secretion and is mitogenic. MC tryptase has the ability to degrade extracellular protein fibronectin, which may contribute to the progress of extracellular tissue destruction [15]. 


\section{Lipid-derived mediators}

The activation of MCs not only causes the release of preformed granule associated mediators, but initiates the de novo synthesis of lipid-derived substances. Of particular importance are the cyclooxygenase (Cox) and lipoxygenase metabolites of arachidonic acid, because these products possess potent inflammatory activity. Cox products include prostaglandins and thromboxanes, whereas lipoxygenases generate LTs, hydroperoxyeicosatetraenoic acids, and the reduced products of the HPETEs, hydroxyeicosatetraenoic acids.

The lipooxygenase pathway forms leukotriene C4 (LTC4), leukotriene D4 (LTD4), and leukotriene E4 (LTE4). Prostaglandin D2 (PGD2) is generated after the immunologic activation of human MCs. PGD2 is a potent inhibitor of platelet aggregation, chemokinetic for human neutrophils and in conjunction with LTD4, it mediates accumulation of neutrophils [15].

\section{Cytokines and chemokines}

MCs produce a number of inflammatory cytokines, including interleukin (IL)-1, IL-2, IL-3, IL-4, IL-5, IL-6, granulocyte-macrophage colony stimulating factor, interferon- $\gamma$ (IFN- $\gamma$ ), and tumor necrosis factor- $\alpha$ (TNF- $\alpha)$ [16].

\section{Mast Cells and Tumor}

The tumor microenvironment is regarded as a 'smoldering' inflammation site in which a lot of cytokines, chemokines and enzymes mediate the inflammatory process and drive malignant progression. Among them TNF- $\alpha$, IL- 6 , vascular endothelial cell growth factor (VEGF), Cox-2, and matrix metalloproteinase-9 (MMP-9) are of particular interest. Coincidentally, all of them can be produced by MCs. However, the tumor microenvironment is also characterized by its immunoediting from immunosurveillance to immunosuppression. MCs have been found to play a critical role in the suppression of immune reactions. They not only produce inhibitory cytokine IL-10, but they also are essential for the immune tolerance mediated by regulatory $\mathrm{T}$ (Treg) cells. Thus, MC infiltration into tumor may possibly remodel tumor microenvironment and profoundly influence tumor behaviour by participating and regulating inflammatory and immune reactions. Studies have shown that MCs also promote tumor angiogenesis and tumor growth because of their properties as inflammatory cells [18].

These findings provide an insight into the role of MCs in tumors and relation among inflammation, immunosuppression and tumor microenvironment.

\section{Role of Mast Cells in Tumor Growth}

MCs appear to be able to promote tumor development through many different ways: they could facilitate tumor angiogenesis through heparin-like molecules and heparin could further permit neovascularisation and metastases through its anti-clotting effects. Moreover, VEGF; vascular permeability factor is secreted in response to $\mathrm{F}_{\mathrm{C}} \varepsilon \mathrm{RI}$ cross linking from mouse bone marrow-derived and human cultured MCs, as well as from human leukemic MCs. MCs also generate and secrete IL-8, which is an angiogenesis factor as well as a tumor cell chemotactic factor and tumor mitogen. MCs secrete growth factors, such as platelet derived growth factor, SCF, and nerve growth factor. They also secrete histamine that could induce tumor cell proliferation through $\mathrm{H}_{1}$ receptors identified in human malignant carcinoma, while suppressing the immune system through $\mathrm{H}_{2}$ receptors [19].

The role of MCs in shaping immune responses has been recently unveiled. MCs may mobilize and modulate the activity of T cells, Treg cells, antigen-presenting cells and myeloid-derived suppressor cells with their soluble mediators or through cell-cell contact. Interacting with other immune cells, MC may regulate both innate and adaptive immunity, tuning the host responses toward developing cancers and influencing the clinical outcome of several tumors [20].

Dimitriadou and Koutsilieris [21] by their pooled data tried to assess the possible association between MCs and tumour progression. They suggested two hypotheses. The first refers on the possibility that the accumulation of MCs is part of a general immunological host-defence reaction since MCs have been shown to be cytotoxic for some tumours. They also considered a second possibility, in which, MC products could promote tumoural growth and metastasis. Heparin, combined to a range of heparin-binding factors such as basic fibroblastic growth factor (bFGF) or transforming growth factor beta (TGF beta), is able to promote neovascularisation and the MC proteases cause cell structural alterations and loss of the extracellular matrix integrity. The role of histamine secreted by MCs is less clear.

\section{Role of Mast Cells in Tumor Angiogenesis}

Kessler et al. [22] observed a strong association between 
tumor angiogenesis factor induced neovascularisation and increased MCD.

Norrby [23] hypothesized about mechanisms of MC mediated angiogenesis, which explained the mechanism being potent and of long duration. MC mediated angiogenesis is initiated by preformed MC mediators such as histamine and TNF- $\alpha$ and stimulated by newly generated MC mediators such as TNF- $\alpha$, platelet activating factor, IL-8, bFGF and prostaglandins. Cytokines such as TNF- $\alpha$ and IL- 8 produced by resident non-MCs (e.g., endothelial cells, fibroblasts and macrophages) and recruited non-MCs may stimulate additional MC secretion as well as stimulate angiogenesis themselves. The activated resident and recruited non-MCs may gradually produce extra cellular matrix degrading enzymes as well as angiogenic peptide growth factors/cytokines. He, thus concluded that MC mediated angiogenesis could be further advanced, potentiated and prolonged.

Coussens et al. [24] conducted an experiment on mice that were genetically MC deficient. The vascular density and architecture in hyperplastic epithelium of MC deficient mice was more characteristic of a quiescent vasculature and suggested that MC-derived factors, including chymase and tryptase, are involved in the early events of neoplastic progression. They concluded that in squamous carcinogenesis angiogenic regulation is biphasic. MCs are exploited by neoplastic epithelia in early lesions and act to jump-start angiogenesis by their release of several bioactive molecules, e.g. bFGF, VEGF, heparin, histamine, chymase, and tryptase. In contrast, maintenance of neovascularization within tumor stroma is MC-independent.

A positive correlation between the $\mathrm{MC}$ and tumor angiogenesis was found in patients with eosophageal squamous cell carcinoma by Tomita et al. [25], invasive breast carcinoma by Kwon et al. [26], patients with gastric carcinoma by Ribatti et al. [27], oral squamous cell carcinoma by Iamaroon et al. [28], and in lung carcinoma by Tomita et al. [29].

\section{Role of Mast Cells in Degradation of Extracellular Matrix}

MCs play an accessory role in the degradation of extracellular matrix, the first of a series of linked sequential steps for a tumor to establish successful metastasis.

Rojas et al. [30] on the basis of their pooled data stated that MC contain chymase which is known for its ability to promote extracellular matrix degradation and for indirectly stimulating angiogenesis. These responses are essential for tumor invasion and metastasis. Chymase activates latent MMPs, including gelatinase B and pro-collagenases, which degrade components of epithelial basement membranes and extracellular matrix, respectively. Other potent extracellular matrix degrading enzymes of MCs involve cathepsin G, carboxypeptidase and the most frequently discovered gelatinase $\mathrm{A}$ and $\mathrm{B}$, which are mediators of tumor progression and metastasis.

Other MC mediators such as FGF-2, TGF- $\beta$, IL-3, and IL- 4 can stimulate collagenase and $\beta$-hexosaminidase production by fibroblasts and IL-1 by macrophages. These factors work in concert to loosen up the stromal milieu to facilitate tumor invasion [2].

\section{Anti Tumor Activity of Mast Cells}

The body of evidence presented thus far supports a tumorigenic role for MCs in the development and progression of malignant lesion. But this can be an incomplete portrayal of the MCs. In fact MCs can oppose the growth of the tumor depending on the microenvironment in which they reside. This dual role for MCs certainly seems probable.

This dual role of MCs is probably because of two reasons. Firstly, MCs have a vast array of mediators, some of which have promoting and others have inhibitory effects on malignancies. Secondly, the phenotypic expression of MC is not static and its secretory pattern alters according to the microenvironment. MCs have the ability to secrete individual granules (in contrast to indiscriminate degranulation in an anaphylactic reaction) or distinct mediators selectively. Several studies have shown a tumor cytotoxic role for MCs in malignant lesions [2].

According to the data pooled by Ozdemir [31] MC mediated cytotoxicity seems to be operated by at least 2 pathways: by secretory pathways via exocytosis of granules containing serine proteases such as granzymes, chymase and soluble TNF- $\alpha$; and nonsecretory (cell-to-cell contact) pathways via membranous TNF- $\alpha$ and FasL.

MCs may promote tumour development through many different ways. On the other hand, MCs could also be detrimental to tumour growth by secreting several cytokines and proteolytic enzymes participating in inducing apoptosis of the malignant cells. The dual role of MCs in inhibiting or promoting tumour growth needs to be further investigated [32]. 


\section{Prognostic Relevance of Mast Cells}

Studies conducted by various researchers have shown that MCD is a useful prognostic marker and is associated with poor prognosis in oral carcinoma [28], pulmonary adenocarcinoma [33], melanoma [34], hepatocellular carcinoma [35], colorectal carcinoma [38]. Tan et al. [37] in their study observed increased MCD to be associated with improved prognosis in colorectal cancer patients. No correlation was found between $\mathrm{MC}$ and the prognosis in invasive breast carcionoma [32]. Also search for novel agents for MC stabilization potential is ongoing [38].

\section{Conclusion}

The studies conducted in animal models have shown that, in the design of drugs for treatment or prevention of cancer or precancerous lesions, it may be beneficial to consider agents that stabilize, restore or improve functionality of MCs. Focusing on the design of MC stabilizers, for example, using a combination of inhibitors of IgE-Fc receptor function, cromolyn derivatives, antihistamines or inhibitors of heparin may prove to be a superior strategy for enhancing the tumoricidal role of MCs. The design and use of suitable MC stabilizers is suggested to reduce MC 'leakiness' and provide an opportunity for MCs to mature (be granulated) for their tumoricidal properties [39].

Therefore, studies suggest MCs as a novel therapeutic target for cancer treatment.

\section{ORCID}

Shikha Saxena: https://orcid.org/0000-0002-3655-1096

Anil Singh: https://orcid.org/0000-0001-6151-2868

Priyanka Singh: https://orcid.org/0000-0002-1616-1867

\section{Author Contributions}

Conceptualization: SS, AS. Data acquisition: SS, PS. Data analysis or interpretation: SS, PS. Drafting of the manuscript: SS. Critical revision of the manuscript: SS, AS. Approval of the final version of the manuscript: all authors.

\section{Conflicts of Interest}

No potential conflict of interest relevant to this article was reported.

\section{References}

1. Walsh LJ. Mast cells and oral inflammation. Crit Rev Oral Biol Med 2003;14:188-98.

2. Ch'ng S, Wallis RA, Yuan L, Davis PF, Tan ST. Mast cells and cutaneous malignancies. Mod Pathol 2006;19:149-59.

3. Elpek GO, Gelen T, Aksoy NH, Erdoğan A, Dertsiz L, Demircan A, Keleş N. The prognostic relevance of angiogenesis and mast cells in squamous cell carcinoma of the oesophagus. J Clin Pathol 2001;54:940-4.

4. Galli SJ. New concepts about the mast cell. N Engl J Med 1993;328:257-65.

5. Galli SJ, Maurer M, Lantz CS. Mast cells as sentinels of innate immunity. Curr Opin Immunol 1999;11:53-9.

6. Grootens J, Ungerstedt JS, Nilsson G, Dahlin JS. Deciphering the differentiation trajectory from hematopoietic stem cells to mast cells. Blood Adv 2018;2:2273-81.

7. Dahlin JS, Hallgren J. Mast cell progenitors: origin, development and migration to tissues. Mol Immunol 2015;63:9-17.

8. Sawai N, Koike K, Mwamtemi HH, Kinoshita T, Kurokawa Y, Sakashita K, Higuchi T, Takeuchi K, Shiohara M, Kamijo T, Ito S, Kato T, Miyazaki H, Yamashita T, Komiyama A. Thrombopoietin augments stem cell factor-dependent growth of human mast cells from bone marrow multipotential hematopoietic progenitors. Blood 1999;93:3703-12.

9. Okayama Y, Kawakami T. Development, migration, and survival of mast cells. Immunol Res 2006;34:97-115.

10. Joseph S, Das S, Chand R, Roopa R, Thomas IM. Comparison of toluidine blue Vs thionin for mast cells in rat mesentery using Carnoy's fixative. J Anat Soc India 2003;52:166-7.

11. Moon TC, Befus AD, Kulka M. Mast cell mediators: their differential release and the secretory pathways involved. Front Immunol 2014;5:569.

12. Wernersson S, Pejler G. Mast cell secretory granules: armed for battle. Nat Rev Immunol 2014;14:478-94.

13. Yamasaki S, Ishikawa E, Kohno M, Saito T. The quantity and duration of FcR $\gamma$ signals determine mast cell degranulation and survival. Blood 2004;103:3093-101.

14. Krishnaswamy G, Kelley J, Johnson D, Youngberg G, Stone W, Huang SK, Bieber J, Chi DS. The human mast cell: functions in physiology and disease. Front Biosci 2001;6:D1109-27.

15. Metcalfe DD, Baram D, Mekori YA. Mast cells. Physiol Rev 1997;77:1033-79.

16. Pejler G, Knight SD, Henningsson F, Wernersson S. Novel insights into the biological function of mast cell carboxypeptidase A. Trends Immunol 2009;30:401-8.

17. Caughey GH. Mast cell tryptases and chymases in inflammation and host defense. Immunol Rev 2007;217:141-54.

18. Huang B, Lei Z, Zhang GM, Li D, Song C, Li B, Liu Y, Yuan Y, Unkeless J, Xiong H, Feng ZH. SCF-mediated mast cell infiltration and activation exacerbate the inflammation and immuno- 
suppression in tumor microenvironment. Blood 2008;112:126979 .

19. Theoharides TC, Conti P. Mast cells: the Jekyll and Hyde of tumor growth. Trends Immunol 2004;25:235-41.

20. Rigoni A, Colombo MP, Pucillo C. The role of mast cells in molding the tumor microenvironment. Cancer Microenviron 2015;8:167-76.

21. Dimitriadou V, Koutsilieris M. Mast cell-tumor cell interactions: for or against tumour growth and metastasis? Anticancer Res 1997;17:1541-9.

22. Kessler DA, Langer RS, Pless NA, Folkman J. Mast cells and tumor angiogenesis. Int J Cancer 1976;18:703-9.

23. Norrby K. Mast cells and de novo angiogenesis: angiogenic capability of individual mast-cell mediators such as histamine, TNF, IL-8 and bFGF. Inflamm Res 1997;46(Suppl 1):7-8.

24. Coussens LM, Raymond WW, Bergers G, Webster LM, Behrendtsen O, Werb Z, Caughey GH, Hanahan D. Inflammatory mast cells up-regulate angiogenesis during squamous epithelial carcinogenesis. Genes Dev 1999;13:1382-97.

25. Tomita M, Matsuzaki Y, Edagawa M, Shimizu T, Hara M, Sekiya R, Onitsuka T. Association of mast cells with tumor angiogenesis in esophageal squamous cell carcinoma. Dis Esophagus 2001;14:135-8.

26. Kwon GY, Lee SD, Park ES. Mast cell and macrophage counts and microvessel density in invasive breast carcinoma-comparison analysis with clinicopathological parameters. Cancer Res Treat 2005;37:103-8.

27. Ribatti D, Guidolin D, Marzullo A, Nico B, Annese T, Benagiano V, Crivellato E. Mast cells and angiogenesis in gastric carcinoma. Int J Exp Pathol 2010;91:350-6.

28. Iamaroon A, Pongsiriwet S, Jittidecharaks S, Pattanaporn K, Prapayasatok S, Wanachantararak S. Increase of mast cells and tumor angiogenesis in oral squamous cell carcinoma. J Oral Pathol Med 2003;32:195-9.

29. Tomita M, Matsuzaki Y, Onitsuka T. Effect of mast cells on tumor angiogenesis in lung cancer. Ann Thorac Surg 2000;69:1686-90.

30. Rojas IG, Spencer ML, Martínez A, Maurelia MA, Rudolph MI. Characterization of mast cell subpopulations in lip cancer. J Oral Pathol Med 2005;34:268-73.

31. Ozdemir O. Immunosurveillance function of human mast cell? World J Gastroenterol 2005;11:7054-6.

32. Amini RM, Aaltonen K, Nevanlinna H, Carvalho R, Salonen L, Heikkilä P, Blomqvist C. Mast cells and eosinophils in invasive breast carcinoma. BMC Cancer 2007;7:165.

33. Takanami I, Takeuchi K, Naruke M. Mast cell density is associated with angiogenesis and poor prognosis in pulmonary adenocarcinoma. Cancer 2000;88:2686-92.

34. Ribatti D, Ennas MG, Vacca A, Ferreli F, Nico B, Orru S, Sirigu P. Tumor vascularity and tryptase-positive mast cells correlate with a poor prognosis in melanoma. Eur J Clin Invest 2003;33:420-5.

35. Peng SH, Deng H, Yang JF, Xie PP, Li C, Li H, Feng DY. Significance and relationship between infiltrating inflammatory cell and tumor angiogenesis in hepatocellular carcinoma tissues. World J Gastroenterol 2005;11:6521-4.

36. Yodavudh S, Tangjitgamol S, Puangsa-art S. Prognostic significance of microvessel density and mast cell density for the survival of Thai patients with primary colorectal cancer. J Med Assoc Thai 2008;91:723-32.

37. Tan SY, Fan Y, Luo HS, Shen ZX, Guo Y, Zhao LJ. Prognostic significance of cell infiltrations of immunosurveillance in colorectal cancer. World J Gastroenterol 2005;11:1210-4.

38. Kandhare AD, Aswar UM, Mohan V, Thakurdesai PA. Ameliorative effects of type-A procyanidins polyphenols from cinnamon bark in compound 48/80-induced mast cell degranulation. Anat Cell Biol 2017;50:275-83.

39. Khatami M. Cyclooxygenase inhibitor ketorolac or mast cell stabilizers: immunologic challenges in cancer therapy. Clin Cancer Res 2005;11:1349-51; author reply 1351. 